Technical note

\title{
Segmentation accuracy of long bones
}

\author{
Joyce Van den Broeck ${ }^{\mathrm{a}, \mathrm{b}, *}$, Evie Vereecke ${ }^{\mathrm{c}}$, Roel Wirix-Speetjens ${ }^{\mathrm{b}}$, Jos Vander Sloten ${ }^{\mathrm{a}}$ \\ a KU Leuven, Biomechanics Section, Celestijnenlaan 300C, 3001 Leuven, Belgium \\ ${ }^{\mathrm{b}}$ Materialise NV, Technologielaan 15, 3001 Leuven, Belgium \\ ${ }^{c}$ KU Leuven, Department of Development E' Regeneration @ Kulak, Etienne Sabbelaan 53, 8500 Kortrijk, Belgium
}

\section{A R T I C L E I N F O}

\section{Article history:}

Received 12 June 2013

Received in revised form 19 March 2014

Accepted 25 March 2014

\section{Keywords:}

Three-dimensional imaging

Imaging accuracy

Medical imaging

Orthopaedics

\begin{abstract}
A B S T R A C T
The use of three-dimensional imaging methodologies in new applications in the orthopaedic field has introduced a need for high accuracy, in addition to a correct diagnosis. The aim of this study was to quantify the absolute dimensional errors between models reconstructed from computed tomography and magnetic resonance images compared to a ground truth for various regions of the bone. Clinical CT and MRI scans were acquired from nine lower leg cadavers and the bones were subsequently cleaned from soft tissues. 3D models of the tibia were created from the segmented CT and MRI images and compared to optical scans of the cleaned bones (considered as ground truth). The 3D reconstruction using CT images resulted in an RMS error of $0.55 \mathrm{~mm}$, corresponding to an overestimated CT bone model compared to the cleaned bone. MR imaging resulted in an RMS error of $0.56 \mathrm{~mm}$; however, the MRI bone model was on average a small underestimation of the cleaned bone. Different regions of the bones were analysed, indicating a difference in accuracy between diaphysis and epiphysis. This study demonstrates a high accuracy for both CT and MRI imaging, supporting the feasibility of using MRI technology for the 3D reconstruction of bones in medical applications.
\end{abstract}

(C) 2014 IPEM. Published by Elsevier Ltd. All rights reserved.

\section{Introduction}

Over the past few years, medical imaging technology has moved from being used for purely diagnostic purposes to a methodology that includes many applications such as patient-specific instrumentation and implants, statistical modelling or joint kinematics research. Computed tomography (CT) or magnetic resonance imaging (MRI) data have both been used to create a three-dimensional (3D) model of the patient's anatomy.

In case of patient-specific instrumentation, 3D models of the patient's anatomy are used to define a preoperative plan [1] and, more recently, for the development of patient-specific implants as well $[2,3]$. Another application that merits from 3D imaging technologies is statistical shape modelling. The availability of 3D bone models of a large number of the population has created an opportunity for both anthropological research and implant companies, as statistical analysis can be performed to map the anatomical variations in a large number of people [4,5]. Patient-specific anatomical models are also applied in research studying the motion of joints.

\footnotetext{
* Corresponding author at: Materialise NV, Technologielaan 15, 3001 Leuven, Belgium. Tel.: +32 167449 30; fax: +32 16396606 .

E-mail address: joyce.vandenbroeck@materialise.be (J. Van den Broeck).
}

Both Lenaerts et al. [6] and Scheys et al. [7] have reported on the importance of patient-specific geometry in musculoskeletal modelling used in gait analysis.

This broad range of applications all have one important requirement in common: the 3D models created from the medical images have to represent an accurate reconstruction of the actual anatomy. In response, a number of studies have been conducted to investigate the dimensional accuracy of 3D models reconstructed using MRI and CT scanning. A limitation to a number of studies performed so far is the lack of a ground truth. Both Lee et al. [8] and Mora-oka et al. [9] investigated the difference between CT and MRI scanning looking at the dimensional accuracy of bone models, without quantifying the errors made with respect to the dimensions of the actual bones. More recently, White et al. [10] and Rathnayaka et al. [11] have reported on studies comparing CT- and MRI-based 3D models to the actual bone, yet their data led to conflicting results. White et al. [10] reported on MRI imaging to result in a very inaccurate representation of the bony anatomy compared to CT imaging; whereas the results presented by Rathnayaka et al. [11] indicate that both MRI and CT imaging result in an accurate 3D model of the bone. The discrepancy in reported accuracy might be due to the different methodology used by these researchers. Rathnayaka et al. [11] performed 3D distance measurements using a point to point comparison method; White et al. [10] produced physical models 


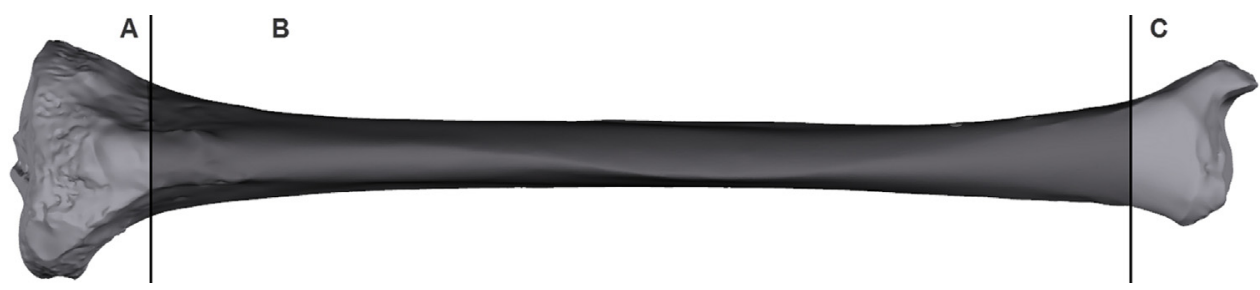

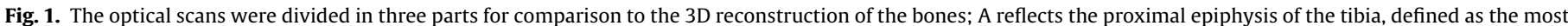
proximal $10 \%$ of the bone, B the diaphyseal part and C the distal epiphysis, identified as the most distal $10 \%$ of the bone.

using 3D printing and compared distance measurements between anatomical landmarks using a calliper.

The study we have conducted, investigates the dimensional accuracy of 3D reconstructions from both MRI and CT data compared to the dimensions of the actual bone, quantified by an optical scan of cleaned bone tissue. In contrast to the previous data available in literature, human cadavers were scanned using a clinical imaging protocol, such that the results give an indication of the feasible accuracy of 3D bone reconstruction in a clinical setting.

\section{Methods}

Ten fresh-frozen legs from six human cadavers (average age: 70 years, age range: 52-92 years, 3 female and 3 male) obtained via the Human Body Donation programme of the University were used to compare different imaging modalities to an optical scan. The legs were amputated mid-femur, preserving the full length of the tibia. Only nine out of ten legs were used for the analysis, as one had to be discarded due to mislabelling during imaging. The specimens were cleaned in two stages: (1) a first cleaning procedure consisted of a mechanical cleaning using standard dissection equipment (i.e. scalpels, scrapers) and immersion of the bones in acetone; afterwards (2) the bones were boiled using a fat-removal procedure [12]. This two-step cleaning procedure ensured the full removal of cartilage and soft tissue.

The cadaveric legs were scanned using both CT and MRI. The MRI images were obtained with a $1.5 \mathrm{~T}$ clinical MRI scanner (GE Signa HDxt) with a torso flex coil using a 3D FAST TOF-SPGR sequence and sagittal slices, with $T R=20 \mathrm{~s}, \mathrm{TE}=5 \mathrm{~s}$, flip angle $=12^{\circ}$, slice thickness $=2 \mathrm{~mm}$, slice increment $=1 \mathrm{~mm}$ and pixel size $=0.39 \mathrm{~mm} \times 0.39 \mathrm{~mm}$. The field of view of the MRI scan covered mainly the knee joint, as would be done in a clinical setting. CT scanning was performed using a clinical CT scanner (GE LightSpeed VCT) using axial slices, $120 \mathrm{kVp}, 160 \mathrm{mAs}$, slice thickness $=0.625 \mathrm{~mm}$ and pixel size $=0.39 \mathrm{~mm} \times 0.39 \mathrm{~mm}$. The scan covered the full tibia. Two CT scans were taken, one of the untreated specimens and one after the first cleaning procedure to quantify the effect of the acetone treatment. MRI scans were only taken of untreated specimens. Specimens were thawed at room temperature $24 \mathrm{~h}$ prior to scanning.

The cleaned bones were optically scanned using a calibrated, white-light optical scanner (ATOS II by GOM mbH, Braunschweig, Germany) with a resolution of 1.2 million pixels per measuring volume, yielding an accuracy of $0.02 \mathrm{~mm}$. This scanner consists of two cameras and a projector unit, which projects a fringe pattern on the object. This pattern is recorded by the cameras and the $3 \mathrm{D}$ shape of the object is obtained via triangulation calculations. By using reference points, different scans are combined together until the complete 3D shape of the object is captured. The optical scan of each bone is considered to reflect the actual dimensions of the bone and acts as ground truth measurement in this study. An optical scan was taken before and after boiling of the bones to quantify the effect of this process on the bone surface and volume.
Manual segmentation of the images was done using Mimics ${ }^{\circledR}$ (v13.3, Materialise NV, Leuven, Belgium). The masks were created by grey value thresholding. Manual mask-based adaptations were applied where necessary. These include for example cropping the mask to the bone of interest, disconnecting the femur and tibia mask if in contact or completing the mask in regions of the images with little contrast and unclear tissue delineation. 3D models were then reconstructed using the following reconstruction settings in Mimics: interpolation method 'gray value', preferred 'accuracy', shell reduction to 1 , no matrix reduction applied and smoothing factor 0.5 using 7 iterations. After reconstruction, the 3D models were exported.

To compare the MRI- and CT-based 3D models to each other and to the optical scans, the models were registered in two steps: an initial registration by aligning the inertia axes and a global ICP-based registration [13]. The optical scan was compared to the imagebased 3D model of the bone by calculating the distance of the vertices of the optical scan to the reconstructed 3D objects, where a positive distance indicates that the optical scan is larger than the image-based model. From this set of error measurements, different metrics were calculated: the mean error and standard deviation, the mean and standard deviation of the absolute, unsigned errors, the root mean square error and the $95 \%$ value of the absolute, unsigned error. The 95\% value represents a good estimation on the overall geometric accuracy of the 3D model and ignores possible outliers in the dataset. After registration, the optical scans were also divided in three parts to compare different regions to the 3D models, as shown in Fig. 1. The aim was to investigate whether the different geometry of diaphyseal and epiphyseal regions of the bones resulted in a different accuracy of reconstructed 3D models. Due to the limited size of the knee coil, the MRI 3D model consisted only of the proximal epiphysis and proximal part of the diaphysis. The models resulting from the different CT scans were also compared to assess the influence of the tissue removal protocol on the superficial bone layer.

\section{Results}

\subsection{Determining the bone-soft tissue interface}

The 3D models generated from the CT scans before and after soft-tissue removal were compared to quantify the impact of the acetone treatment. The results, listed in Table 1 , indicate that the bone model reconstructed after treatment is on average smaller than the CT-scan based reconstruction of the untreated bone. This error was introduced by the cleaning process that removes part of the bone - soft tissue interface (illustrated in Fig. 2). To remove remaining ligament attachments, the bones were boiled in a second cleaning step. This procedure has been previously reported to have an additional shrinking effect on the bone interface [12]. This effect is quantified by comparing the optical scans of the bones before and after the boiling process. As can be seen in Table 1, the optical scan of the bone after boiling was smaller than the optical scan before boiling. 
Table 1

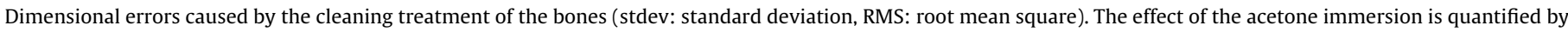

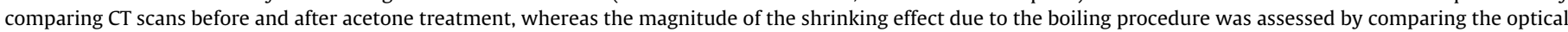
scans before and after boiling the bones. A positive value indicates that the model before cleaning is larger than the model after cleaning.

\begin{tabular}{|c|c|c|c|c|}
\hline Cleaning procedure & Absolute error: Mean (Stdev) [mm] & Signed error: Mean (Stdev) [mm] & RMS error [mm] & Absolute error: $95 \%$ value $[\mathrm{mm}]$ \\
\hline Acetone immersion & $0.40(0.52)$ & $0.25(0.61)$ & 0.66 & 1.97 \\
\hline Boiling procedure & $0.20(0.13)$ & $0.16(0.18)$ & 0.24 & 0.46 \\
\hline Total error & $0.43(0.52)$ & $0.40(0.53)$ & 0.67 & 1.81 \\
\hline
\end{tabular}

\subsection{Imaging accuracy}

The results of comparing the 3D models of the CT images of the untreated legs and the optical scans of the fully cleaned bones are listed in Table 2. The average error is negative which indicates that on average the CT-based 3D bone model is an overestimation of the actual bone, represented by the optical scan. In addition, the diaphysis of the bone is reconstructed with a higher accuracy compared to the epiphyseal regions. Fig. 3 provides an example of the comparison between the CT-based bone model and the optical scan.

The MRI-based 3D bone models were compared to the optical scans of the cleaned bones and the results are presented in Table 3. The average error comparing MRI segmentation and optical scan is positive, indicating that an MRI-based bone reconstruction gives an underestimation of the dimensions of the actual bone. The epiphysis of the bone is reconstructed with a higher accuracy compared to the diaphysis. However, the reconstructed proximal epiphysis represents on average a small overestimation of the cleaned bone while the reconstructed diaphysis is an underestimation of the cleaned bone. The comparison between the optical scan and MRIbased bone reconstruction is illustrated in Fig. 4.

\subsection{Overall results}

Combining the results from the comparison of the CT- and MRIbased 3D bone models to the optical scans of the actual bone and the tests to investigate the impact of removing the bone-soft tissue interface allows us to quantify the overall accuracy of the imaging methodologies in a clinical setting. As indicated in Table 2, 3D bone models created from CT images will be an overestimation of the actual bone, even when taking into account the impact of the cleaning error, resulting in a shrinking of the actual bone. The MRI segmentation results in an opposite situation: the 3D bone model will be on average an underestimation of the cleaned bone (as indicated in Table 3), and an underestimation of the actual bone as well.

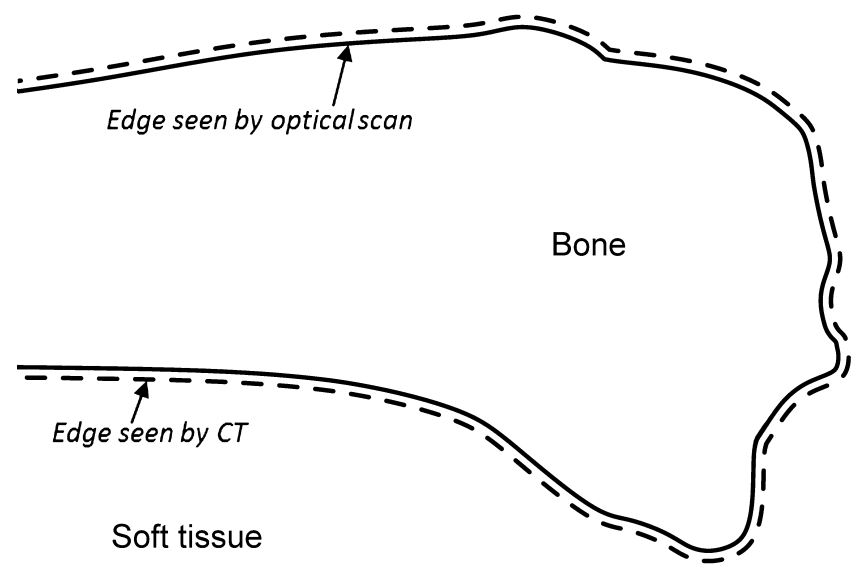

Fig. 2. Illustration of the bone - soft tissue interface situated between the delineation of the actual bone (full line) and the border with the soft tissues visible on CT images (dotted line).

\section{Conclusions}

This study has investigated the accuracy of segmenting long bones using a clinical imaging protocol. It was found that both CT and MRI imaging are accurate for 3D bone reconstructions within $0.5 \mathrm{~mm}$ compared to the ground truth. On average, CT segmentation will result in a slight overestimation compared to the actual dimensions of the bone whereas MRI segmentation will induce a small underestimation of the bone's geometry.

The results reported on can be compared to previous studies investigating imaging accuracy. White performed a study looking at CT and MRI images to quantify the accuracy for the design of patient-specific instrumentation for total knee arthroplasty [10]. An average accuracy of $0.61 \mathrm{~mm}$ on CT reconstruction and $2.15 \mathrm{~mm}$ using MRI images was found. However, the accuracy of the reconstruction was assessed by 3D printing of physical bone models and measuring the distances between landmark points using a calliper. As high inter-user variability has been reported on the identification of anatomical landmarks and part of their reported inaccuracy could be caused by the measuring protocol $[14,15]$.

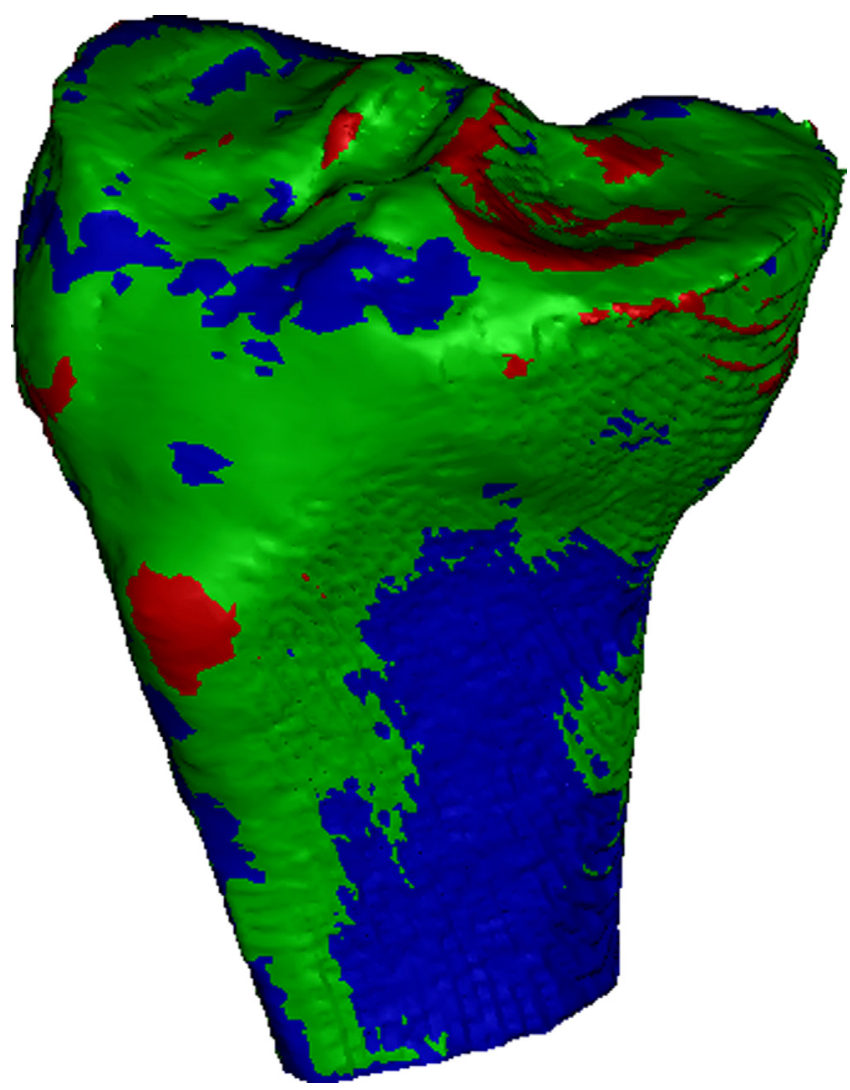

Fig. 3. Illustration of a typical result comparing the CT-based bone model with the optical scan of the bone. The difference is within $0.5 \mathrm{~mm}$ in the green regions and below $-0.5 \mathrm{~mm}$ in the red regions. A negative value (red) indicates that the $3 \mathrm{D}$ model is an overestimation of the actual bone. (For interpretation of the references to colour in this figure legend, the reader is referred to the web version of this article.) 
Table 2

Difference between the 3D models reconstructed from the segmentation of the CT scan of the intact leg and the optical scan of the cleaned bone.

\begin{tabular}{|c|c|c|c|c|}
\hline Bone region & Absolute error: Mean (Stdev) [mm] & Signed error: Mean (Stdev) [mm] & RMS error [mm] & Absolute error: $95 \%$ value [mm] \\
\hline Proximal epiphysis & $0.58(0.26)$ & $-0.57(0.27)$ & 0.64 & 0.96 \\
\hline Diaphysis & $0.48(0.14)$ & $-0.48(0.14)$ & 0.50 & 0.67 \\
\hline Distal epiphysis & $0.54(0.37)$ & $-0.50(0.42)$ & 0.65 & 1.17 \\
\hline Full bone & $0.51(0.22)$ & $-0.50(0.23)$ & 0.55 & 0.88 \\
\hline
\end{tabular}

Table 3

Difference between the 3D model from the MRI segmentation of the intact leg and the optical scan after boiling of the bones.

\begin{tabular}{|c|c|c|c|c|}
\hline Bone region & Absolute error: Mean (Stdev) [mm] & Signed error: Mean (Stdev) [mm] & RMS error [mm] & Absolute error: $95 \%$ value $[\mathrm{mm}]$ \\
\hline Proximal epiphysis & $0.39(0.37)$ & $-0.07(0.53)$ & 0.58 & 1.14 \\
\hline Diaphysis & $0.46(0.36)$ & $0.20(0.55)$ & 0.54 & 1.14 \\
\hline Full bone & $0.41(0.37)$ & $0.04(0.55)$ & 0.56 & 1.14 \\
\hline
\end{tabular}

Rathnayaka compared both CT- and MRI-based 3D models to measurements of the actual bone using a mechanical contact scanner [11]. An absolute accuracy of $0.15 \mathrm{~mm}$ for CT and $0.23 \mathrm{~mm}$ for MRI was found, using five ovine limbs, where CT-based 3D models resulted in a slight overestimation and MRI-based models in an underestimation of the actual bone. The results from our study are close to the ones reported on by Rathnayaka. Differences in the results may be attributed to a difference in imaging protocol and image processing technique.

Our study setup was designed to be similar to the study performed by Gelaude on the accuracy of CT imaging [12]. In their study, CT reconstructions of the femur before and after soft-tissue removal were compared to a ground truth, created by a mechanical

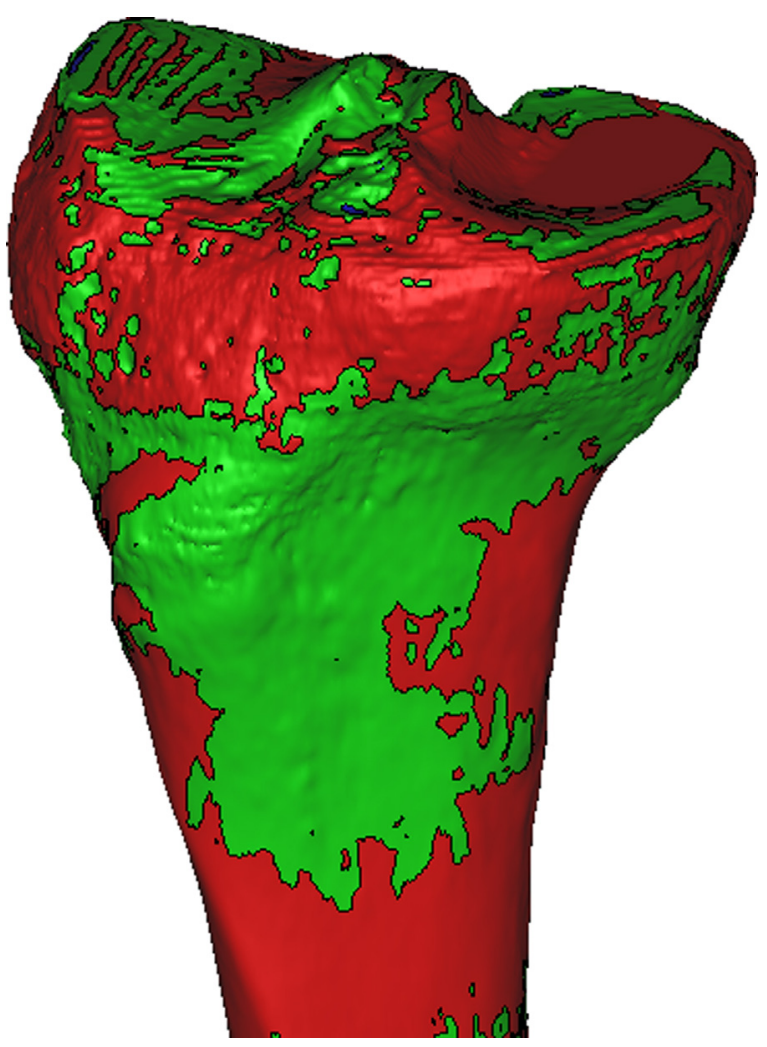

Fig. 4. Illustration of a typical result comparing the MRI-based bone model with the optical scan of the bone. The difference is within $0.5 \mathrm{~mm}$ in the green regions, below $-0.5 \mathrm{~mm}$ in the red regions and above $0.5 \mathrm{~mm}$ in the blue regions. A positive value (blue) indicates that the 3D model is an underestimation of the actual bone. (For interpretation of the references to colour in this figure legend, the reader is referred to the web version of this article.) contact scan of the bones, to quantify both the CT error and the error created by the soft-tissue removal process. The error reported on by comparing the CT-based reconstruction with the actual bone is similar to the one found in this study, as is the RMS error.

As the boiling procedure has been reported to result in shrinking of the bones [12], in this study an acetone treatment was applied initially to avoid or limit this additional error. However, as the acetone treatment was not sufficient to remove all ligament attachments, a subsequent boiling procedure was applied. The combination of these two cleaning procedures did not result in large dimensional changes to the bone or bone interface; the resulting cleaning error was comparable to the one reported by Gelaude et al. [12], who applied just a boiling procedure.

When looking at the reconstruction accuracy of the different bone regions, different results were found for CT- and MRI-based bone models. Using CT data, the diaphysis of the bone is reconstructed more accurately than the epiphyses. This can be explained by the higher grey value gradient present because of the thicker cortical bone, resulting in a more straightforward image segmentation compared to the epiphysis where the contrast is less. The opposite is true for MRI data: as the knee joint is aligned in the centre of the MRI coil, the signal is weaker towards the extremities resulting in a lesser grey value gradient and more difficult image segmentation. In addition, the surrounding cartilage and joint fluid at the epiphysis provides a clear delineation of the bone, where this border is less visible in the diaphyseal regions with the current imaging protocol. This likely explains the higher accuracy for the proximal epiphysis compared to the diaphysis.

This study has demonstrated the feasibility of 3D reconstruction of bones using MRI technology with an accuracy comparable to CT imaging, and hence the feasibility of using MRI technology for preoperative planning or the design of patient-specific implants or instrumentation in orthopaedic or craniomaxillofacial surgery.

\section{Acknowledgements}

Joyce Van den Broeck acknowledges the Baekeland Scheme of the Agency for Innovation by Science and Technology (IWT, grant number: IWT090732) for financial support. The authors also wish to thank Elise Lesage, Jo Verbinnen and Kristof Reyniers for technical support in data collection. We also wish to thank the anonymous reviewers for their constructive feedback on the original manuscript.

\section{Funding}

Financial support was received from the Baekeland scheme of the agency for Innovation by Science and Technology (IWT).

\section{Conflicts of interest}

The authors declare no competing interests. 


\section{Ethical approval}

Not required.

\section{References}

[1] Dobbe JGG, Du Pré KJ, Kloen P, Blankevoort L, Streekstra GJ. Computerassisted and patient-specific 3-D planning and evaluation of a single-cut rotational osteotomy for complex long-bone deformities. Med Biol Eng Comput 2011;49:1363-70.

[2] Harrysson OLA, Hosni YA, Nayfeh JF. Custom-designed orthopedic implants evaluated using finite element analysis of patient-specific computed tomography data: femoral-component case study. BMC Musculos Disord 2007; 8:91.

[3] Koeck FX, Beckmann J, Luring C, Rath B, Grifka J, Basad E. Evaluation of implant position and knee alignment after patient-specific unicompartmental knee arthroplasty. Knee 2011;18:294-9.

[4] Gillespie RJ, Levine A, Fitzgerald SJ, Kolaczko J, DeMaio M, Marcus RE, et al. Gender differences in the anatomy of the distal femur. J Bone Joint Surg Br Vol 2011:93:357-63.

[5] Waarsing JH, Rozendaal RM, Verhaar JAN, Bierma-Zeinstra SMA, Weinans H. A statistical model of shape and density of the proximal femur in relation to radiological and clinical OA of the hip. Osteoarthr Cartil 2010;18:787-94.

[6] Lenaerts G, Bartels W, Gelaude F, Mulier M, Spaepen A, Van der Perre G, et al. Subject-specific hip geometry and hip joint centre location affects calculated contact forces at the hip during gait. J Biomech 2009;42:1246-51.
[7] Scheys L, Van Campenhout A, Spaepen A, Suetens P, Jonkers I. Personalized MR-based musculoskeletal models compared to rescaled generic models in the presence of increased femoral anteversion: effect on hip moment arm lengths. Gait Posture 2008;28:358-65.

[8] Lee YS, Seon JK, Shin VI, Kim G-H, Jeon M. Anatomical evaluation of CT-MRI combined femoral model. Biomed Eng Online 2008;7:6.

[9] Moro-oka T, Hamai S, Miura H, Shimoto T, Higaki H, Fregly BJ, et al. Can magnetic resonance imaging-derived bone models be used for accurate motion measurement with single-plane three-dimensional shape registration? J Orthop Res 2007;8:7-72.

[10] White D, Chelule KL, Seedhom BB. Accuracy of MRI vs CT imaging with particular reference to patient specific templates for total knee replacement surgery. Int J Med Robot Comput Assist Surg 2008;4:224-31.

[11] Rathnayaka K, Momot KI, Noser H, Volp A, Schuetz M.a., Sahama T, et al. Quantification of the accuracy of MRI generated 3D models of long bones compared to CT generated 3D models. Med Eng Phys 2012;34:357-63.

[12] Gelaude F, Vander Sloten J, Lauwers B. Accuracy assessment of CT-based outer surface femur meshes. Comput Aided Surg 2008;13:188-99.

[13] Besl PJ, McKay ND. A method for registration of 3D shapes. IEEE Trans Pattern Anal Mach Intell 1992;14:239-56.

[14] Perrin N, Stindel E, Roux C. BoneMorphing versus freehand localization of anatomical landmarks: consequences for the reproducibility of implant positioning in total knee arthroplasty. Comput Aided Surg 2005;10: 301-9.

[15] Robinson M, Eckhoff DG, Reinig KD, Bagur MM, Bach JM. Variability of landmark identification in total knee arthroplasty. Clin Orthop 2006;442: $57-62$ 